\title{
A Novel Online Scheduling Algorithm for Hierarchical Vehicle-to-Grid System
}

\author{
Xiangyu Chen, Ka-Cheong Leung, Albert Y. S. Lam, and David J. Hill \\ Department of Electrical and Electronic Engineering \\ The University of Hong Kong \\ Pokfulam Road, Hong Kong, China \\ E-mail:\{xychen, kcleung, ayslam, dhill\}@eee.hku.hk
}

\begin{abstract}
In recent years, the vehicle-to-grid (V2G) system, which utilizes electric vehicles (EVs) to provide ancillary services for power grid, draws a lot of interests in smart grid research community. When considering a large number of EVs distributed in different geographical locations, how to coordinate these EVs to provide ancillary services becomes a critical issue. In this paper, a generic hierarchical framework for V2G system to provide frequency regulation services is proposed to address this issue. A practical multi-level online V2G algorithm is proposed for the hierarchical V2G scheduling and it requires no forecasting information for regulation signals. We test our proposed algorithm in the simulation of a four-level hierarchical V2G system. The results show that the proposed algorithm has advantages over the existing methods on smoothing out the real-time power fluctuations.
\end{abstract}

\section{INTRODUCTION}

There is a trend that electric vehicles (EVs) will take a significant market share in transportation in the near future. Due to the increasing popularity of EVs, the research community has shown interest in studying the interaction between the EVs and the power grid. Some recent studies have shown that the power electronics and batteries inside EVs can respond well to frequency regulation signals through battery charging and discharging [1]-[3]. Therefore, an aggregation of EVs, which constitutes the vehicle-to-grid (V2G) system as distributed energy storage, can bring huge capacities for regulation services. It is expected that EVs can be excellent candidates for providing frequency regulation services by balancing power in the grid [4].

When it comes to a large number of EVs, their charging and discharging behaviors must be coordinated so as to satisfy the regulation requirement. Without proper coordination and control, the stochastic EV charging and discharging behaviors can bring uncertainty to the grid power balance and jeopardizes the stability and reliability of the grid. Therefore, control and scheduling for EV charging/discharging are important research topics in recent years. Existing research on the control for V2G regulation services includes: the frequency deviation approach and the optimization approach. The former employs droop characteristics to drive the frequency deviation from the regulated frequency to zero [5]-[7] and it allows EVs to respond quickly to power fluctuations without communication [5] or with very little communication [6], [7]. However, since this approach only relies on the frequency signal which lacks sophisticated EV coordination schemes, it is unable to achieve the global optimal for $\mathrm{V} 2 \mathrm{G}$ regulation services.

For the optimization approach, a global optimization problem is formulated to guide the charging and discharging behaviors of EVs toward the global optimum. In [8]-[11], One aggregator was deployed to coordinate the EV fleet to provide regulation services. [9] proposed a distributed EV coordination mechanism, which manages a fleet of EVs to offer V2G regulation services based on a day-ahead scheduling profile. [10] proposed a welfare-maximizing regulation allocation algorithm for the aggregator to allocate the $\mathrm{V} 2 \mathrm{G}$ regulation power fairly among the EVs. In [11], a game-theoretic model was proposed to understand the interactions among EVs and aggregators in a V2G market.

When managing a large-scale V2G system with operators at different levels of operations and EVs at different geographical locations, one aggregator is not sufficient to oversee the whole system. In recent years, the hierarchical architecture was studied for large-scale V2G system to coordinate a large number of EVs distributed over a large geographical area [12], [13]. The algorithms proposed in [12] and [13] were designed for three-level V2G systems with bi-level programming models. The authors in [12] proposed a hierarchical charging control framework based on the Benders decomposition for a large population of EVs. [13] proposed a hierarchical decomposition approach to coordinate the charging and discharging behaviors of plug-in EVs. The designed V2G structures in [12] and [13] are not extensible as the number of levels of the V2G system is restricted.

Inspired by [14], in this paper, a generic framework for hierarchical V2G system is proposed. The proposed hierarchical V2G framework is extensible as there is no limit on the number of levels in the V2G system. The contributions of this work are two-fold. First, we develop the concept of smart V2G aggregator (SVA) to coordinate the charging and discharging of subordinate EVs in the intermediate levels and the detailed protocols among the grid operator, SVAs, and EVs, are implemented. Second, an algorithm for hierarchical V2G scheduling is devised. To the best of our knowledge, neither theory nor algorithm has been developed for generic hierarchical V2G scheduling. We show that our proposed multi- 


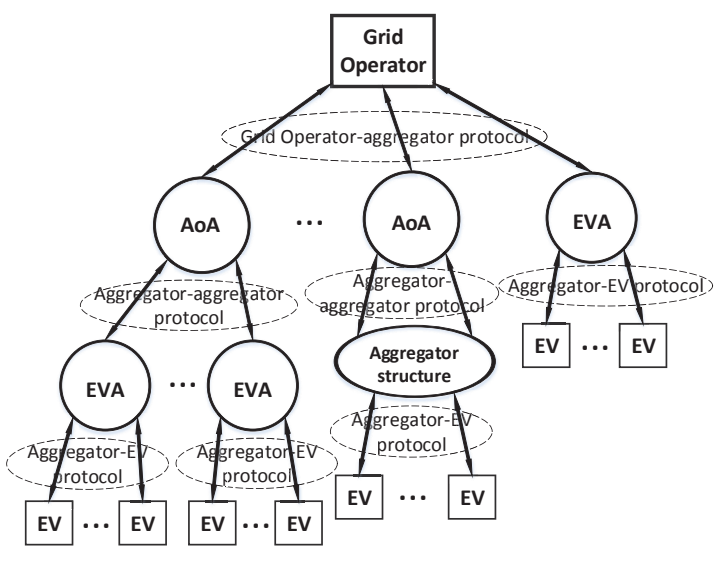

Fig. 1. System Model.

level online V2G (MLOV) algorithm can respond well to the regulation signals and does not require any prediction information for regulation requests, which is different from the forecasting-based approaches developed in [8]-[13].

The rest of this paper is organized as follows. The system model is introduced in Section II. Section III formulates optimization framework for the power assignment problem. The MLOV algorithm is devised for the operation of hierarchical V2G system in Section IV. The simulation results of the proposed algorithm are examined in Section $\mathrm{V}$ and the paper is concluded in Section VI.

\section{SYSTEM MODEL}

\section{A. System Architecture}

The multi-level V2G system is deployed to provide frequency regulation services for the power grid through balancing the grid power by coordinating the charging and discharging of EVs. The multi-level V2G system architecture, shown in Fig. 1, consists of three key components: the grid operator, a set of aggregators, and a fleet of EVs. It has a hierarchical structure with multiple levels of nodes. The utility grid operator, SVAs, and EVs correspond to the root, intermediate, and leaf nodes, respectively. We further categorize SVAs into two types; those directly connected to EVs are denoted as EV aggregators (EVAs) while the others are called aggregators of aggregators (AoAs). The size of a SVA is determined by its subordinate EV fleets with other geographical, economic, and technical factors. For instance, a parking lot or a charging station can be configured with an EVA. Several SVAs can be further managed by an AoA. In this model, each node can only communicate with its neighboring nodes in the tree. For example, the grid operator can only communicate with those SVAs in the first level. Each EVA can only communicate with its parent AoA and its subordinate EVs. Be noted that, in this paper, all the flows between adjacent nodes in the tree are information flows.

\section{B. Flows of Information protocols}

As illustrated in Fig. 1, three types of operation protocols can be defined. By the nature of the communicating parties,

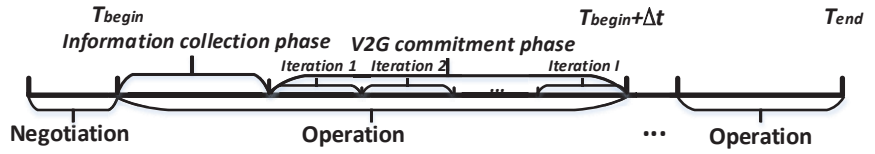

Fig. 2. Operation Timeline.

they can be referred to the grid operator-aggregator protocol, the aggregator-aggregator protocol, and the aggregatorEV protocol. Each protocol operates between a node and its immediate subordinate nodes. The V2G scheduling has two phases, namely, the information collection phase and the V2G commitment phase:

1) Information collection phase: In the hierarchical V2G scheduling, to assign reasonable and feasible $\mathrm{V} 2 \mathrm{G}$ power signals to the child nodes, a parent node should collect the V2G capacities from its child nodes, which is a bottom-totop process and will be discussed in Section III-C. The V2G capacity of a certain node indicates how much power it can provide to the power grid for regulation service. Specifically, the V2G capacity of an EV refers to its V2G power limits, based on its state-of-charge (SOC), the charging requirement, and its maximum charging and discharging rates. The $\mathrm{V} 2 \mathrm{G}$ capacity of a SVA corresponds to the current V2G power limits of this SVA, i.e., the aggregated V2G power limits of all its subordinate EVs.

2) V2G commitment phase: After the information collection phase, the V2G commitment phase assigns the V2G power signals in the top-down manner to meet the grid regulation request, which is formulated as the power assignment problem analyzed in Section III-D. A lower level then returns the commitment signal of providing regulation services to an upper level. This process repeats iteratively until the V2G commitment process converges at each level.

The timeline for the whole operation of hierarchical $V 2 \mathrm{G}$ system is illustrated as Fig. 2.

\section{Basic Models}

Consider that the multi-level V2G system provides regulation service over the time horizon $\left[T_{\text {begin }}, T_{\text {end }}\right]$, which is divided equally into $T$ time slots, each of which has a duration of $\Delta t$ minutes.

The architecture of the multi-level V2G system has $K$ levels, each of which is labeled with $k \in\{1,2, \ldots, K\}$. In the information collection phase at time slot $t$, the estimated V2G capacity for the $m$ th node in the $k$ th level is denoted as $[\underline{A}(k, m, t), \bar{A}(k, m, t)]$, where $\underline{A}(k, m, t)$ and $\bar{A}(k, m, t)$ are the lower and upper power limits of $\mathrm{V} 2 \mathrm{G}$ power in $\mathrm{kW}$, respectively. Let $R_{g}(t) \mathrm{kW}$ be the regulation request of grid operator at $t, R(k, m, t) \mathrm{kW}$ and $P(k, m, t) \mathrm{kW}$ be the assigned share of the regulation request and assigned $\mathrm{V} 2 \mathrm{G}$ power for the $m$ th node in the $k$ th level at time slot $t$, respectively. Note that $R(k, m, t)$ is generally not equal to $P(k, m, t)$. The relationship between $R(k, m, t)$ and $P(k, m, t)$ will be explained in Section III-D.

Consider that in the $(K-1)$ th level, a specific EVA coordinates $N$ EVs to schedule their charging or discharging powers. 
EV $n$ plugs in at the beginning of the time slot $t_{n, i n}$ with its initial SOC, denoted by $S_{n, i n}$, where $n \in\{1,2, \ldots, N\}$. The SOC of EV $n$ at the beginning of the time slot $t$ is given as $S_{n}(t) \in\left[\underline{S}_{n}, \bar{S}_{n}\right]$, where $\bar{S}_{n}$ and $\underline{S}_{n}$ indicate its upper and lower bounds, respectively. The departure time for EV $n$ is at the beginning of the time slot $t_{n, \text { out }}$. Each EV $n$ should satisfy the charging requirement before departure and thus $S_{n}\left(t_{n, \text { out }}\right) \geq S_{n, \text { req }}$ should hold. The charging or discharging rate of $\mathrm{EV} n$ at time slot $t$ is denoted as $P_{n}(t) \mathrm{kW}$, with maximum charging limit $\bar{P}_{n} \mathrm{~kW}$ and discharging limit $\underline{P}_{n} \mathrm{~kW}$.

Without loss of generality, we adopt the following assumptions:

1) The EVs are available for negotiation at the beginning of the scheduling period.

2) The regulation service cannot be predicted and is a zero-energy service, which means the expectation of the energy required for regulation service is zero over a long time period.

The first assumption is made in many previous related works [8]-[13]. For the second assumption, it is reasonable since frequency regulation requests arise from the forecasting errors of grid power [15].

\section{PROBLEM Formulation}

In this section, we first introduce the control objective and constraints for the $\mathrm{V} 2 \mathrm{G}$ scheduling problem. Then, we analyze the calculation of $\mathrm{V} 2 \mathrm{G}$ capacity in the information collection phase. Finally, the power assignment problem in V2G commitment phase is formulated.

\section{A. Control Objective for V2G Regulation Services}

When providing regulation services with $\mathrm{V} 2 \mathrm{G}$, we aim to minimize the variation of the total power, which is the summation of the regulation request and the V2G power. In other words, the objective is to smooth out the power fluctuations of the grid due to the power imbalance in the grid.

Based on the control objective, the following objective function is formulated for each root and intermediate node, including the grid operator and SVAs. Consider that a parent node $o$ in $k$ th level has $M$ child nodes denoted by a set $\mathcal{M}_{o}=\{1,2, \ldots, M\}$. The parent node $o$ assigns the shares of V2G power $P\left(k+1, \mathcal{M}_{o}, t\right):=(P(k+1,1, t), P(k+$ $1,2, t), \ldots, P(k+1, M, t))$ to its child nodes $m \in \mathcal{M}_{o}$. The objective function is formulated as follows:

$$
\begin{aligned}
& F\left(R(k, o, t), P\left(k+1, \mathcal{M}_{o}, t\right)\right)=\operatorname{Var}\left(P_{\text {total }}(\mathcal{T})\right) \\
= & \left.\frac{1}{T} \sum_{i=1}^{T}\left(P_{\text {total }}(i)-\frac{1}{T}\left(\sum_{j=1}^{T} P_{\text {total }}(j)\right)\right)\right)^{2}
\end{aligned}
$$

where $\operatorname{Var}(\cdot)$ denotes the function for calculating variance, the total power of time $i$ is denoted as $P_{\text {total }}(i)=R(k, o, i)+$ $\sum_{m \in \mathcal{M}_{o}} P\left(k+1, \mathcal{M}_{o}, i\right), R(k, o, i)$ is the assigned share of regulation request of time $i$ for parent node, and $P(k+1, m, i)$ is the assigned $\mathrm{V} 2 \mathrm{G}$ power of time $i$ for child node $m \in \mathcal{M}_{o}$.

\section{B. Operational Constraints}

Consider that a specific EVA $m$ coordinates $N$ EVs to schedule their charging or discharging. For EV $n, n \in \mathcal{N}_{m} \triangleq$ $\{1,2, \ldots, N\}$, the EV charging and discharging powers $P_{n}(t)$ should follow:

$$
\left\{\begin{array}{cc}
P_{n} \leq P_{n}(t) \leq \overline{P_{n}}, & t \in\left[t_{n, \text { in }}, t_{n, \text { out }}\right) \\
P_{n}(t)=0, & t \notin\left[t_{n, \text { in }}, t_{n, \text { out }}\right) .
\end{array}\right.
$$

(2) means that $P_{n}(t)$ should lie within the maximum charging and discharging limits during the plug-in period, and it is equal to zero when it is not plugged.

Considering the energy efficiency of battery charging and discharging, its SOC $S_{n}(t)$ is updated with:

$S_{n}(t+1)=S_{n}(t)+\eta\left(P_{n}(t)\right) P_{n}(t) \Delta t, \quad t \in\{1,2, \ldots, T-1\}$.

The battery efficiency $\eta(x)$ is defined as:

$$
\eta(x)= \begin{cases}\eta_{c h} & \text { if } x>0, \\ 1 & \text { if } x=0, \\ \frac{1}{\eta_{d c h}} & \text { if } x<0\end{cases}
$$

where $\eta_{c h}$ and $\eta_{d c h}$ denote the charging and discharging efficiencies, respectively.

There are two constraints confining SOC of EV $n$. Firstly, the SOC should lie within the battery's lower and upper limits for the health of the battery:

$$
\underline{S}_{n} \leq S_{n}(t) \leq \bar{S}_{n}, \quad t \in\{1,2, \ldots, T\} .
$$

Secondly, EV $n$ should be charged up to a satisfactory level before its departure:

$$
\begin{aligned}
& S_{n}(t)+\eta\left(P_{n}(t)\right) P_{n}(t) \Delta t+\eta\left(\bar{P}_{n}\right) \bar{P}_{n}\left(t_{n, \text { ou }}-t-1\right) \Delta t \\
& \geq S_{n, \text { req }}+S_{n, S M}(t), \quad t \leq t_{n, \text { out }}-1 .
\end{aligned}
$$

The left-hand side of this inequality denotes the maximum SOC that the EV can be charged, with the charging or discharging power $P_{n}(t)$ at time $t . S_{n, S M}(t)$ is called the "safety margin" [14] of EV $n$ which is defined by:

$$
\begin{aligned}
& S_{n, S M}(t)= \\
& \begin{cases}\mu\left(\bar{S}_{n}-S_{n, \text { req }}\right), & t \in\left[T_{n, \text { in }},(1-\tau) T_{n, \text { in }}+\tau T_{n, \text { out }}\right] \\
0 & \text { otherwise }\end{cases}
\end{aligned}
$$

where $0 \leq \mu \leq 1$ specifies the size of the "safety margin" and $0 \leq \tau \leq 1$ determines the duration the effective period of the "safety margin." By considering $S_{n, S M}(t)$ in (6), the EV can buffer more energy to counter the uncertainty of the regulation request.

For SVAs, the assigned V2G power $P(k, m, t)$ of SVA $m$ in the $k$ th level at time slot $t$ should fall into its V2G capacity:

$$
\underline{A}(k, m, t) \leq P(k, m, t) \leq \bar{A}(k, m, t), \quad t \in \mathcal{T}
$$

where $\underline{A}(k, m, t)$ and $\bar{A}(k, m, t)$ are calculated in Section III-C.

\section{Calculation of $V 2 G$ capacity}

Here we explain how to determine the capacity of an individual EV for $\mathrm{V} 2 \mathrm{G}$ services. Then it is elaborated how the V2G capacity of SVAs and grid operator can be calculated. 
1) V2G Capacity of individual EVs: Combining constraints (2)-(6), EV $n$ can acquire the estimated V2G capacity as:

$$
\begin{aligned}
& \max \left(\underline{P_{n}}, \eta_{d c h} \frac{S_{n}-S_{n}(t)}{\Delta t}, \frac{\gamma}{\Delta t \eta(\gamma)}\right) \leq \\
& P_{n}(t) \leq \min \left(\overline{P_{n}}, \frac{\overline{S_{n}}-S_{n}(t)}{\eta_{c h} \Delta t}\right), \text { if } t \in\left[t_{n, \text { in }}, t_{n, \text { out }}\right)
\end{aligned}
$$

where

$$
\gamma=S_{n, r e q}+S_{n, S M}(t)-S_{n}(t)-\eta_{c h} \overline{P_{n}}\left(t_{n, \text { out }}-t-1\right) \Delta t
$$

and

$$
P_{n}(t)=0, \quad \text { if } t \notin\left[t_{n, \text { in }}, t_{n, \text { out }}\right) .
$$

We denote $\underline{C}_{n}(t)$ and $\bar{C}_{n}(t)$ as the lower and upper limits of $\mathrm{V} 2 \mathrm{G}$ power for $\mathrm{EV} n$ at time slot $t$, respectively. They are specified as follows:

$$
\begin{aligned}
& \underline{C}_{n}(t)= \\
& \left\{\begin{array}{l}
\max \left(\frac{P_{n}}{{ }^{2}}, \eta_{d c h} \frac{S_{n}-S_{n}(t)}{\Delta t}, \frac{\gamma}{\Delta t \eta(\gamma)}\right), \quad t \in\left[t_{n, \text { in }}, t_{n, \text { out }}\right) \\
0, \quad t \notin\left[t_{n, \text { in }}, t_{n, \text { out }}\right)
\end{array}\right. \\
& \quad \bar{C}_{n}(t)= \\
& \quad\left\{\begin{array}{l}
\min \left(\overline{P_{n}}, \frac{\overline{S_{n}}-S_{n}(t)}{\eta_{\text {ch }} \Delta t}\right), \quad t \in\left[t_{n, \text { in }}, t_{n, \text { out }}\right) \\
0, \quad t \notin\left[t_{n, \text { in }}, t_{n, \text { out }}\right)
\end{array}\right.
\end{aligned}
$$

Intuitively, the $\mathrm{V} 2 \mathrm{G}$ capacity of an EV is constrained by its maximum charging and discharging limits, the current storage SOC, and the charging need before departure.

2) V2G Capacity of SVAs and grid operator: After all EVs have determined its V2G capacity, the AEV collects the V2G capacities of its child EVs resulting in the aggregated V2G capacity. For AEV $m$ at the $(K-1)$ th level coordinating EVs in set $\mathcal{N}_{m}$, the aggregated lower and upper limits of V2G power, i.e., $\underline{A}(k, m, t)$ and $\bar{A}(k, m, t)$, are determined by:

$$
\begin{aligned}
& \underline{A}(K-1, m, t)=\sum_{n \in \mathcal{N}_{m}} \underline{C}_{n}(t) \\
& \bar{A}(K-1, m, t)=\sum_{n \in \mathcal{N}_{m}} \bar{C}_{n}(t) .
\end{aligned}
$$

The AoAs and grid operator can calculate their V2G capacities based on the V2G capacities of their child nodes. Consider a parent node $o$ at the $k$ th level, which can be a AoA or the grid operator, supervises $M$ child nodes at the $(k+1)$ th level that are labeled with $m \in\{1,2, \ldots, M\}=\mathcal{M}_{o}$. Each child node has obtained its V2G capacity from its own child nodes, which is expressed by the lower limit $\underline{A}(k+1, m, t)$ and the upper limit $\bar{A}(k+1, m, t)$. The aggregated lower and upper limits of V2G power for $o$, i.e., $\underline{A}(k, o, t)$ and $\bar{A}(k, o, t)$, are calculated by:

$$
\begin{aligned}
& \underline{A}(k, o, t)=\sum_{m \in \mathcal{M}_{o}} \underline{A}(k+1, m, t) \\
& \bar{A}(k, o, t)=\sum_{m \in \mathcal{M}_{o}} \bar{A}(k+1, m, t),
\end{aligned}
$$

for $k \in\{1,2, \ldots, K-2\}$.

\section{Formulation of Power Assignment Problem}

We formulate optimization framework for the power assignment problem in the $\mathrm{V} 2 \mathrm{G}$ commitment phase for each protocol.

1) Grid operator-aggregator protocol and aggregatoraggregator protocol: We design a generic regulation assignment protocol for the grid operator and intermediate SVAs (AoAs). Consider Node $o$ in the $k$ th level, where $k \in$ $\{1,2, \ldots, K-2\}$. The assigned V2G powers on its child node $m \in \mathcal{M}_{o}=\{1,2, \ldots, M\}$, denoted as $P\left(k+1, \mathcal{M}_{o}, t\right):=$ $(P(k+1,1, t), P(k+1,2, t), \ldots, P(k+1, M, t))$, are determined by solving the following optimization problem:

$$
\begin{aligned}
& \underset{P\left(k+1, \mathcal{M}_{o}, t\right)}{\operatorname{minimize}} F\left(R(k, o, t), P\left(k+1, \mathcal{M}_{o}, t\right)\right)+ \\
& \beta \sum_{m \in \mathcal{M}_{o}}\left(P(k+1, m, t)-P_{c o m}(k+1, m, t)\right)^{2}
\end{aligned}
$$

subject to (8) and

$$
|P(k+1, m, t)-\bar{P}(k+1, m, t)| \leq e^{-i \phi(k)}
$$

where $\beta$ is the penalty coefficient for the deviation between the assigned $\mathrm{V} 2 \mathrm{G}$ power and the commitment signal $P_{\text {com }}(k+1, m, t), k \in\{1,2, \ldots, K-2\}$, and $m \in \mathcal{M}_{o}$. Constraint (19) is devised to ensure the convergence of the operation algorithm in Section IV for iteration $i, \bar{P}(k+1, m, t)$ is the assigned $\mathrm{V} 2 \mathrm{G}$ power in the iteration $i-1$, and $\phi(k)$ is the convergence coefficient for level $k$.

After receiving the assigned power $P(k+1, m, t)$, each child node updates its assigned share of regulation request $R(k+1, m, t)$ as follows:

$$
R(k+1, m, t)=-\left(P(k+1, m, t)-\frac{P_{r e q}(k+1, m)}{T}\right)
$$

where $P_{r e q}(k+1, m)=\sum_{n \in m}\left(S_{n, r e q}-S_{n, i n}\right)$ is the total EV charging needs of SVA $m$ in the $(k+1)$ th level. $P_{\text {req }}(k+1, m)$ is a fixed value and can be obtained at the EV negotiation period. Including $P_{r e q}(k+1, m)$ in (20) aims at satisfying the $\mathrm{EV}$ charging needs when EVs offer regulation services.

2) Aggregator-EV protocol: In the $(K-1)$ th level, EVA coordinates the charging or discharging behaviors of its EVs based on the designed aggregator-EV protocol. Consider an EVA $m$ which coordinates EVs in set $\mathcal{N}_{m}$ to provide regulation services, the assigned EV charging or discharging power $P_{n}(t)$ for $\mathrm{EV} n \in \mathcal{N}_{m}=\{1,2, \ldots, N\}$ is determined based on the following optimization problem:

$$
\begin{aligned}
& \underset{P_{\mathcal{N}_{m}}(t)}{\operatorname{minimize}} F\left(R(K-1, m, t), P_{\mathcal{N}_{m}}(t)\right) \\
& \text { subject to (2), (3), (5), and (6). }
\end{aligned}
$$

where $P_{\mathcal{N}_{m}}(t):=\left(P_{1}(t), P_{2}(t), \ldots, P_{N}(t)\right)$.

For problems (18) and (21), $P(k+1, m, i)$ and $P_{n}(i)$ become future decision variables when $i>t$ holds. This makes the problems (18) and (21) unable to be solved directly. We can approximate (1) so that the control objective only includes current decision variables $P(k+1, m, t)$ and $P_{n}(t)$. Therefore, 
the convex problems (18) and (21) can be solved easily. Due to the space limitation, the derivation is omitted.

\section{Algorithm for Hierarchical V2G Scheduling}

For hierarchical V2G scheduling, MLOV Algorithm is proposed as Algorithm 1. We assume the number of nodes in the $k$ th level is denoted as $N_{k}$. The set of child nodes for parent node $o$ is denoted as $\mathcal{M}_{o}$. In the beginning of each time slot, the information collection phase is executed in Steps 2-5. In the information collection phase, EVs and SVAs determine their V2G capacities. In Steps 6-18, the V2G commitment phase is executed. In each iteration, the grid operator and SVAs assign the shares of V2G power to their child nodes based on (18) and (21). EVs and SVAs then return the commitment signals for V2G powers in Steps 12-15. The V2G commitment phase is executed iteratively until the algorithm converges, where the conditions in step 17 are satisfied. Assuming each parent node has the same number of child nodes, denoted by $N_{c}$, the computational complexity of MLOV algorithm is $\mathcal{O}\left(K N_{c}\right)$.

The convergence of the MLOV algorithm can be guaranteed by including constraint (19) in the optimization problem (18). In iteration $i$, the deviation of the assigned $\mathrm{V} 2 \mathrm{G}$ powers between iteration $i$ and iteration $i-1$ is bounded by $e^{-i \phi(k)}$. As $i$ increases, the deviation of the assigned shares of $\mathrm{V} 2 \mathrm{G}$ power between two adjacent iterations vanishes for each node in each level, which corresponds to the convergence of the algorithm.

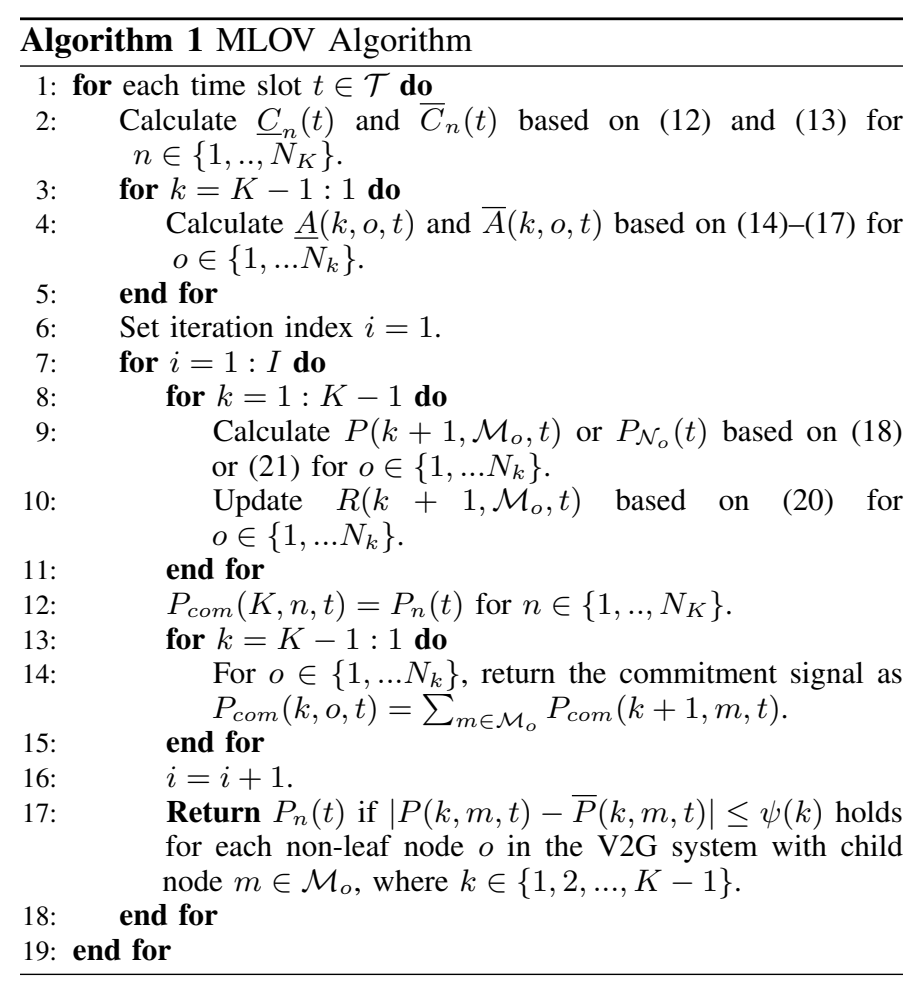

\section{Performance Evaluation}

\section{A. Simulation Setup}

We evaluate the performance of the proposed MLOV algorithm with a four-level hierarchical V2G system, which includes 10, $000 \mathrm{EVs}$ supporting regulation services for a power grid. The grid operator governs ten first-level SVAs, each of which manages ten second-level SVAs correspondingly. Each of these second-level SVA (EVA) then coordinates 100 EVs to determine their charging/discharging behaviors. We consider a time horizon of 1000 minutes (from 07:00 in the morning to 23:40 in the evening), which is divided equally into $T=200$ slots of length $\Delta t=5$ minutes.

The EVs are assumed to be homogeneous ${ }^{1}$ with maximum charging and discharging limits of $2 \mathrm{~kW}$ and $-1 \mathrm{~kW}$, respectively. The battery size of each EV is $20 \mathrm{kWh}$ and both the charging and discharging efficiencies are set to 0.9 . The plugin time of an EV follows a normal distribution with the mean at 08:00 and the standard deviation of 60 minutes. Any plug-in time before 07:00 is set to be 07:00. Similarly, the departure time of an EV is assumed to follow a normal distribution with the mean at 22:00 and the standard deviation of 60 minutes. Any departure time after 23:40 is set be to 23:40. The regulation signals from the PJM market in July 1, 2016 [16] are adopted in our simulation. The standard deviation of the original regulation power $\sigma=2.17 \times 10^{3} \mathrm{~kW}$. In Section V-C1, the regulation power is multiplied by $1.5,2$, and 2.5 times to test the effectiveness of algorithm under different regulation levels. $\mu$ and $\tau$ in (7) are set to be 0.1 and 0.8 , respectively.

\section{B. Scenarios for Comparison}

To the best of our knowledge, there is no existing algorithm for online scheduling for generic hierarchical V2G system. Instead, three algorithms, namely, (S1) the constant charging algorithm, (S2) the greedy charging algorithm based on [5], and (S3) the proposed MLOV algorithm, will be investigated and compared.

In $\mathrm{S} 1$, each $\mathrm{EV}$ is controlled to charge at a fixed rate between the plug-in time and the departure time. S2 is designed based on the grid measurement approach to provide regulation services for the power grid [5]. In S2, the V2G power of each individual $\mathrm{EV}$ is controlled with droop characteristics against the grid frequency deviation. It should be noted that S1 and S2 directly control individual EVs. They can work in the hierarchical V2G system but without the help of aggregators. The performance of the algorithms is evaluated by the variance of the profile of the total power.

\section{Simulation Results}

1) Effectiveness of MLOV Algorithm: The effectiveness of the MLOV algorithm for smoothing out the power grid fluctuations is shown in Fig. 3 for different regulation levels. The dashed curve corresponds to the total regulation request received by grid operator and the solid curve represents the total grid power regulated by $\mathrm{V} 2 \mathrm{G}$ services through the MOLV algorithm. We can see that the proposed MOLV algorithm can smooth out the fluctuations of the regulation requests and maintain the total grid power to a constant level. As the standard deviation of the regulation power $\sigma \mathrm{kW}$ becomes

\footnotetext{
${ }^{1}$ It should be noted that our devised framework is also applicable for heterogeneous EVs.
} 

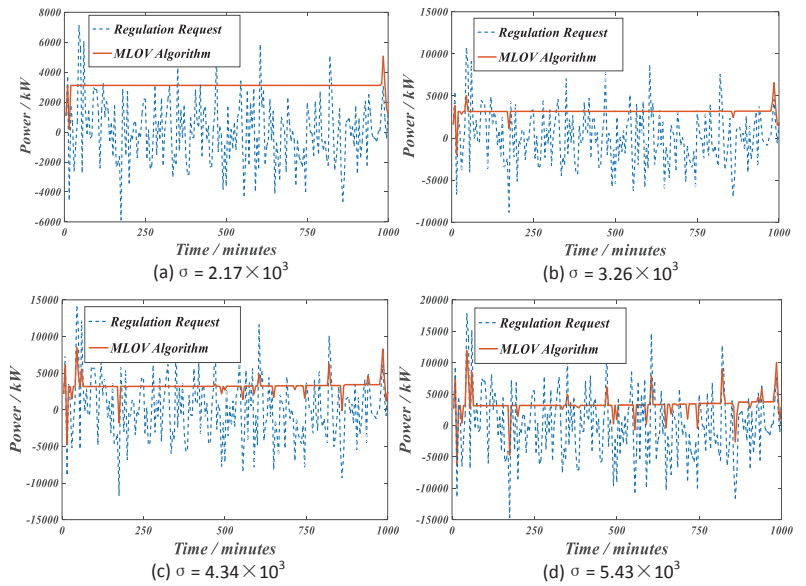

Fig. 3. Effectiveness of MLOV Algorithm under Different Regulation Level.

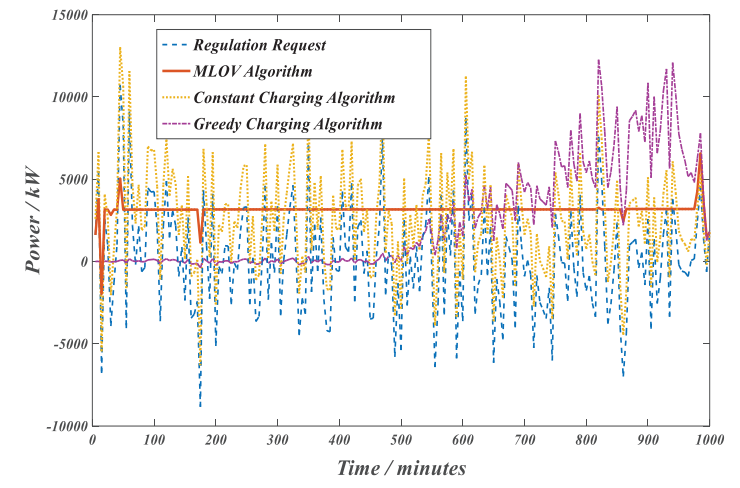

Fig. 4. Comparison among Algorithms.

larger, which means the amplitude of the regulation request increases, the total grid power suffers more and more spikes since the EVs regulation capacities are inadequate to deal with the abrupt changes of the regulation requests.

2) Comparison of Different Algorithms: The comparison of three algorithms for smoothing out the power grid fluctuation is illustrated in Fig. 4. We can conclude that $\mathrm{S} 1$ cannot respond to the regulation signals in which the total grid power suffers from severe fluctuations. S2 can compensate well for the fluctuations of regulation request at the beginning of the scheduling time and then deteriorates since it does not consider the coordination and schedule of EVs in the whole scheduling horizon. When the EVs are about to depart, they have to charge from power grid simultaneously in order to satisfy their own charging needs, thus bring huge burdens to the power grid. The MLOV algorithm can respond well to the regulation requests and is able to maintain the total grid power to a constant level. The fluctuations occur at the beginning and toward the end of the simulation due to the plug-in and the departure processes of EVs.

\section{CONCLUSION}

This paper proposes a generic hierarchical framework for V2G system to coordinate EVs for regulation services. The communication protocols among the grid operator, aggregators, and EVs are well defined. The power assignment optimization problem is formulated to guide the operations of the system. Without relying on any forecasting information of the regulation requests, the proposed MLOV algorithm is very practical for the operations of hierarchical V2G system. Our simulation results show that the MLOV algorithm is able to smooth out the power fluctuations of the grid effectively.

\section{ACKNOWLEDGEMENT}

This research is supported in part by the Research Grants Council of the Hong Kong Special Administrative Region, China, under Grant No. 17261416.

\section{REFERENCES}

[1] C. H. Merrill, V. H. Lam, M. J. V. Vleet, M. S. Chatti, M. C. Brannon, E. B. Connelly, J. H. Lambert, D. L. Slutzky, and J. P. Wheeler, "Modeling and simulation of fleet vehicle batteries for integrated logistics and grid services," in 2015 Systems and Information Engineering Design Symposium, Apr. 2015, pp. 255-260.

[2] O. C. Onar, M. Starke, G. P. Andrews, and R. Jackson, "Modeling, controls, and applications of community energy storage systems with used EV/PHEV batteries," in 2012 IEEE Transportation Electrification Conference and Expo (ITEC), Jun. 2012.

[3] M. Petit and Y. Perez, "Plug-in vehicles for primary frequency regulation: What technical implementation?" in 2013 IEEE Grenoble Conference, Jun. 2013.

[4] A. Y. S. Lam, K.-C. Leung, and V. O. K. Li, "Capacity estimation for vehicle-to-grid frequency regulation services with smart charging mechanism," IEEE Trans. Smart Grid, vol. 7, no. 1, pp. 156-166, Jan. 2016.

[5] Y. Ota, H. Taniguchi, T. Nakajima, K. M. Liyanage, J. Baba, and A. Yokoyama, "Autonomous distributed V2G (Vehicle-to-Grid) satisfying scheduled charging," IEEE Transactions on Smart Grid, vol. 3, no. 1, pp. 559-564, Mar. 2012.

[6] T. N. Pham, H. Trinh, and L. V. Hien, "Load frequency control of power systems with electric vehicles and diverse transmission links using distributed functional observers," IEEE Transactions on Smart Grid, vol. 7, no. 1, pp. 238-252, Jan. 2016.

[7] H. Yang, C. Y. Chung, and J. Zhao, "Application of plug-in Electric Vehicles to frequency regulation based on distributed signal acquisition via limited communication," IEEE Transactions on Power Systems, vol. 28, no. 2, pp. 1017-1026, May 2013.

[8] J. J. Escudero-Garzas, A. Garcia-Armada, and G. Seco-Granados, "Fair design of plug-in electric vehicles aggregator for $\mathrm{V} 2 \mathrm{G}$ regulation," IEEE Transactions on Vehicular Technology, vol. 61, no. 8, pp. 3406-3419, Oct. 2012.

[9] E. L. Karfopoulos, K. A. Panourgias, and N. D. Hatziargyriou, "Distributed coordination of electric vehicles providing $\mathrm{V} 2 \mathrm{G}$ regulation services," IEEE Transactions on Power Systems, vol. 31, no. 4, pp. 2834-2846, Jul. 2016.

[10] S. Sun, M. Dong, and B. Liang, "Real-time welfare-maximizing regulation allocation in dynamic aggregator-EVs system," IEEE Transactions on Smart Grid, vol. 5, no. 3, pp. 1397-1409, May 2014.

[11] C. Wu, H. Mohsenian-Rad, and J. Huang, "Vehicle-to-aggregator interaction game," IEEE Trans. Smart Grid, vol. 3, no. 1, pp. 434-442, Mar. 2012.

[12] C. Shao, X. Wang, X. Wang, C. Du, and B. Wang, "Hierarchical charge control of large populations of EVs," IEEE Transactions on Smart Grid, vol. 7, no. 2, pp. 1147-1155, Mar. 2016.

[13] W. Yao, J. Zhao, F. Wen, Y. Xue, and G. Ledwich, "A hierarchical decomposition approach for coordinated dispatch of plug-in electric vehicles," IEEE Transactions on Power Systems, vol. 28, no. 3, pp. 2768-2778, Aug. 2013.

[14] J. Lin, K.-C. Leung, and V. O. K. Li, "Optimal scheduling with vehicletogrid regulation service," IEEE Internet of Things Journal, vol. 1, no. 6, pp. 556-569, Dec. 2014.

[15] B. J. Kirby, Frequency regulation basics and trends. United States. Department of Energy, 2005.

[16] "Regulation Signal Data," PJM, Aug, 2016. [Online. Available: http://www.pjm.com/-/media/markets-ops/ancillary/mkt-basedregulation/regulation-data.ash $x ? \mathrm{la}=\mathrm{en}]$. 